\title{
Diagnostic and prognostic significance of long noncoding RNA LINC00173 in patients with melanoma
}

\author{
Mujun Wang ${ }^{1} \oplus$, Wei Liu² $\odot$, Wenxing Liu $^{3} \odot$, Chao Wang ${ }^{1 *} \odot$
}

\section{SUMMARY}

OBJECTIVE: A growing volume of literature has suggested long noncoding RNAs (IncRNAs) as important players in tumor progression. In this study, we aimed to investigate the expression and prognostic value of IncRNA LINC00173 (LINC00173) in melanoma.

METHODS: LINC00173 expression was measured in 163 paired cancerous and noncancerous specimen samples by real-time polymerase chain reaction. The correlations between LINC00173 expression with clinicopathological characteristics and prognosis were analyzed by chi-square test, log-rank test, and multivariate survival analysis. Receiver-operating characteristic curves were used for the assessment of the diagnostic value of LINC00173 for melanoma patients.

RESULTS: The expression level of LINC00173 in melanoma specimens was distinctly higher than that in adjacent non-neoplasm specimens ( $<<0.01$ ). Besides, LINC00173 was expressed more frequently in patients with advanced melanoma than in patients with early melanoma. Multivariate assays confirmed that LINC00173 expression level was an independent prognostic predictor of melanoma patients $(p<0.05)$.

CONCLUSION: Our data indicated that LINC00173 expression could serve as an unfavorable prognostic biomarker for melanoma patients. KEYWORDS: Long Noncoding RNA LINC00173. Melanoma. Biomarker.

\section{INTRODUCTION}

Human melanoma is one of the most aggressive and frequently diagnosed cancers in humans ${ }^{1}$. In China, melanoma has displayed an increasing trend ${ }^{2}$. Despite the continued advancements in surgical operation program, radiation, and chemotherapy, the therapeutic effectiveness of advanced melanoma has not shown distinct improvements, and the long-term survivals remain dismal ${ }^{3,4}$. The poor prognosis for melanoma patients was due to the high frequency of metastasis and recurrence 5 . Therefore, it is imperative to reveal the mechanisms underlying melanoma progression and identify new biomarkers and therapeutic targets for melanoma patients.

Long noncoding RNAs (lncRNAs) are another class of noncoding RNA with more than 200 nucleotides in length that lack a complete open reading frame $(\mathrm{ORF})^{6}$. Growing studies have demonstrated that lncRNAs are conserved in many animals and specifically expressed in several cellular types and the development of biological progress ${ }^{7}$. LncRNAs play essential roles in several biological processes, and several different functions of lncRNAs in the gene modulation have been identified, such as protein sponges and miRNAs sponges ${ }^{8,9}$. Importantly, growing studies have focused on the potential effects of lncRNAs in tumor progression via the novel mechanism "miRNAs sponge"10,11. Moreover, the frequently dysregulated expressing pattern of lncRNAs in clinical tumor specimens is positively correlated with malignancy grades and histological differentiation, which suggests that some functional lncRNAs may be used as potential biomarkers in tumor diagnosis of subclassification and prognostication ${ }^{12,13}$. In recent years, several melanoma-related lncRNAs have been functionally identified and could be used as novel prognostic and diagnostic biomarkers for melanoma patients, such as lncRNA HOTAIR, lncRNA PVT1, and lncRNA CRNDE ${ }^{14-16}$.

In this study, we identified a novel melanoma-related lncRNA, i.e., lncRNA LINC00173 (LINC00173), which was first functionally identified in lung cancer by Yang et $\mathrm{al}^{17}$. The distinct upregulation of LINC00173 has been confirmed in several tumors, such as gastric carcinoma, esophagus cancer, and breast cancer ${ }^{18-20}$. However, its expression and biological function in tumors remained largely unclear. For the first time, we provided clinical evidence that LINC00173 was highly expressed in melanoma and may be used as a novel diagnostic and prognostic biomarker for melanoma patients.

\footnotetext{
${ }^{1}$ The First People's Hospital of Jinan City, Department of Surgery - Jinan, China.

${ }^{2}$ The First Affiliated Hospital of Shandong First Medical University, Department of Medical Ultrasound - Jinan, China.

${ }^{3}$ Shizhong District People's Hospital of Jinan, Department of Surgery - Jinan, China.

*Corresponding author: wangchaowc0531@163.com

Conflicts of interest: the authors declare there is no conflicts of interest. Funding: none.

Received on August 22, 2021. Accepted on October 23, 2021.
} 


\section{METHODS}

\section{Patients and tissue samples}

The 163 melanoma tissues and their pair-matched nontumor specimens in this study (collected from May 2012 to July 2015) were obtained from patients who underwent radical resections at the first people's hospital of Jinan city. All cases gave informed consent for their samples to be used for our experiments. None of the patients had received radiotherapy or chemotherapy before surgery. All collected tissue samples were immediately snap-frozen and stored for further real-time polymerase chain reaction (RT-PCR). A comprehensive set of clinical parameters was recorded by the follow-up department of our hospital and further used for clinicopathological and prognostic assays. The Ethics Committee of our hospital approved our study.

\section{RNA extraction and qRT-PCR assays}

With the use of TRIzol reagent (Invitrogen, Hangzhou, Zhejiang, China), the extraction of total RNA from melanoma specimens and matched nontumor tissues was carried out based on the standard procedures provided by the company. A PrimeScript RT Reagent Kit with cDNA eraser (Takara Biotech, Pudong, Shanghai, China) was used for cDNA synthesis with one microgram of total RNA as a template. RT-PCR was carried out using cDNA primers specific for LINC00173 and mRNA. GAPDH was used as an internal control for LINC00173. Primers for LINC00173 were purchased from Genecopoeia (Xuhui, Shanghai, China). LINC00173: Forward GCCAGCTCTCGGTACCTGGA, LINC00173: Reverse GGATCGCAACATTCCTGCCAAG; GAPDH: ForwardCAAGGTCATCCATGACAACTTTG, GAPDH: ReverseGTCCACCACCCTGTTGCTGTAG.
The relative expression of LINC00173 was expressed as $\Delta \mathrm{Ct}=\mathrm{Ct}$ gene $-\mathrm{Ct}$ reference, and the $2^{-\Delta \Delta \mathrm{Ct}}$ methods were applied for the calculation of the fold change.

\section{Statistical analysis}

All statistical analyses were conducted using SPSS version 16.0 software (SPSS Inc., Chicago, IL, USA). The difference between two independent groups was compared by an independent sample t-test. The chi-square test was used to analyze the relationship between categorical variables. Receiver-operating characteristic (ROC) curve assays were applied to determine the sensitivity and specificity of LINC00173 for melanoma diagnosis. The Kaplan-Meier method was performed for patients' overall survival (OS) and disease-free survival (DFS) to identify independent prognostic factors of significance, and a univariate and multivariate Cox regression was performed. The results were considered to be statistically significant at $\mathrm{p}<0.05$.

\section{RESULTS}

\section{The differential expression of LINC00173 in melanoma specimens}

To determine the expression of LINC00173 in melanoma, qRT-PCR was performed by using fresh melanoma tissues. As presented in Figure 1A, we observed that the expression levels of LINC00173 were distinctly increased in human melanoma specimens compared with adjacent nontumor tissues ( $\mathrm{p}<0.01)$. Besides, LINC00173 was shown to be expressed more frequently in advanced melanoma patients than that in early melanoma patients $(\mathrm{p}<0.01)$. Our findings indicated LINC00173 as a regulator in melanoma progression.

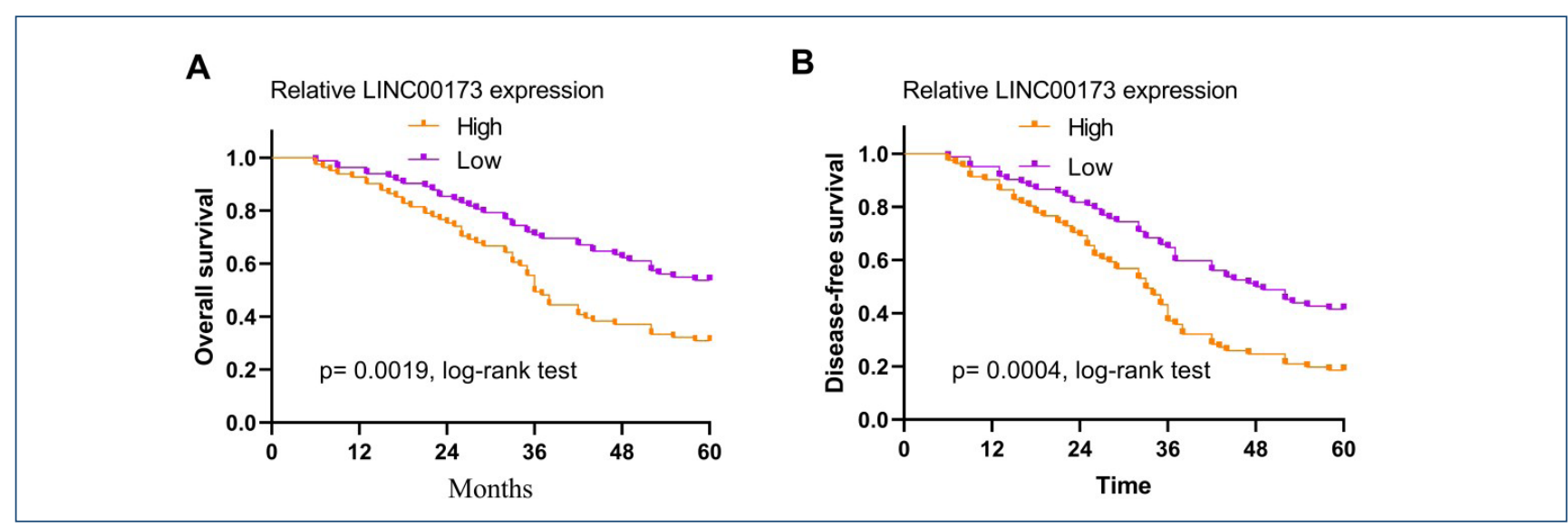

Figure 1. Kaplan-Meier curves of the overall survival (A) and disease-free survival (B) of 163 melanoma patients based on the expression of LINC00173. 


\section{Diagnostic value of LINC00173 overexpression in melanoma}

To examine the characteristics of LINC00173 as a potential diagnostic biomarker for melanoma patients, ROC curves were constructed on data from all subjects. The results revealed that the area under the curve (AUC) of ROC curve was 0.7695 (95\%CI: 0.7172-0.8217, $\mathrm{p}<0.001$, sensitivity: 0.76 , specificity: 0.85 ). Besides, we also performed subgroup analysis, finding that there was a strong separation between the patients with advanced stages and patients with early stages, with an AUC of 0.7451 (95\%CI: 0.6512-0.8389; $\mathrm{p}<0.001)$ for LINC00173. Overall, our findings indicated LINC00173 as a potential diagnostic biomarker for melanoma patients.

\section{Expression levels of LINC00173 and clinicopathological parameters in melanoma}

To investigate the possible significance of LINC00173 expression in clinical progress, all 163 patients were divided into low LINC00173 expression group and high LINC00173 expression group based on the median LINC00173 level in all melanoma specimens. The data from a chi-square test revealed that high LINC00173 expression was positively associated with advanced tumor stage $(\mathrm{p}=0.026)$ and lymph node metastasis $(\mathrm{p}=0.007)$ (Table 1). However, there was no difference in age and sex $(\mathrm{p}>0.05)$.

\section{High expression of LINC00173 correlates with poor prognoses}

During the follow-up, 94 patients died, and the median follow-up time was $37 \pm 12.3$ months. Then, we performed KaplanMeier analysis to explore the prognostic value of LINC00173 in melanoma patients. Interestingly, patients with higher LINC00173 expression have shown significantly lower OS $(p=0.0019$, Figure $1 A)$ and DFS $(p=0.0004$, Figure $1 B)$ than those with lower LINC00173 expression. To further examine the probability of LINC00173 expression as an independent prognostic parameter of patient outcomes, univariate and multivariate assays were performed. We observed that high LINC00173 expression (OS: $\mathrm{p}=0.014$, DFS: $\mathrm{p}=0.017$, Table 2 ) was an independent prognostic parameter, indicating poor clinical outcome for melanoma patients.

Table 1. Correlation between LINC00173 expression and clinicopathological characteristics of melanoma patients.

\begin{tabular}{|c|c|c|c|c|}
\hline \multirow{2}{*}{ Clinicopathological features } & \multirow{2}{*}{ No. of cases } & \multicolumn{2}{|c|}{ LINC00173 expression } & \multirow{2}{*}{ p-value } \\
\hline & & High & Low & \\
\hline \multicolumn{5}{|l|}{ Age (years) } \\
\hline$<55$ & 79 & 35 & 44 & \multirow{2}{*}{0.182} \\
\hline$\geq 55$ & 84 & 46 & 38 & \\
\hline \multicolumn{5}{|l|}{ Sex } \\
\hline Male & 92 & 50 & 42 & \multirow{2}{*}{0.176} \\
\hline Female & 71 & 31 & 40 & \\
\hline \multicolumn{5}{|l|}{ Tumor thickness (mm) } \\
\hline$\leq 2$ & 106 & 47 & 59 & \multirow{2}{*}{0.062} \\
\hline$>2$ & 57 & 34 & 23 & \\
\hline \multicolumn{5}{|l|}{ Ulceration } \\
\hline- & 110 & 51 & 59 & \multirow{2}{*}{0.121} \\
\hline+ & 53 & 30 & 23 & \\
\hline \multicolumn{5}{|l|}{ Tumor stage } \\
\hline I/II & 110 & 48 & 62 & \multirow{2}{*}{0.026} \\
\hline III/IV & 53 & 33 & 20 & \\
\hline \multicolumn{5}{|l|}{ Lymph node metastasis } \\
\hline Negative & 120 & 52 & 68 & \multirow{2}{*}{0.007} \\
\hline Positive & 43 & 29 & 14 & \\
\hline
\end{tabular}


Table 2. Prognostic factors for overall survival or disease-free survival by univariate and multivariate analysis.

\begin{tabular}{|c|c|c|c|c|}
\hline \multirow{2}{*}{ Variables } & \multicolumn{2}{|c|}{ Univariate analysis } & \multicolumn{2}{|c|}{ Multivariate analysis } \\
\hline & $\mathrm{HR}(95 \% \mathrm{Cl})$ & p-value & $\mathrm{HR}(95 \% \mathrm{Cl})$ & p-value \\
\hline \multicolumn{5}{|l|}{ Overall survival } \\
\hline Age & $0.985(0.472-1.885)$ & 0.217 & - & - \\
\hline Sex & $1.217(0.665-2.128)$ & 0.185 & - & - \\
\hline Tumor thickness & $1.467(0.885-2.109)$ & 0.118 & - & - \\
\hline Ulceration & $1.378(0.839-1.885)$ & 0.121 & & \\
\hline Tumor stage & $2.923(1.327-4.772)$ & 0.013 & $2.652(1.175-4.429)$ & 0.018 \\
\hline Lymph node metastasis & $3.451(1.482-5.428)$ & 0.001 & $3.176(1.285-4.995)$ & 0.009 \\
\hline LINC00173 expression & $2.986(1.328-4.774)$ & 0.009 & $2.768(1.157-4.456)$ & 0.014 \\
\hline \multicolumn{5}{|l|}{ Disease-free survival } \\
\hline Age & $1.275(0.687-1.984)$ & 0.218 & - & - \\
\hline Sex & $0.857(0.478-2.187)$ & 0.182 & - & - \\
\hline Tumor thickness & $1.447(0.852-2.217)$ & 0.118 & - & - \\
\hline Ulceration & $1.522(0.832-2.143)$ & 0.105 & & \\
\hline Tumor stage & $1.445(1.442-4.342)$ & 0.009 & $1.352(1.322-4.195)$ & 0.015 \\
\hline Lymph node metastasis & $3.321(1.372-5.127)$ & 0.002 & $3.018(1.138-4.726)$ & 0.009 \\
\hline LINC00173 expression & $2.938(1.278-4.662)$ & 0.006 & $2.629(1.158-4.385)$ & 0.017 \\
\hline
\end{tabular}

\section{DISCUSSION}

Melanoma is one of the most frightful human diseases in both developed and developing countries, largely due to its high-grade malignancy, fast infiltrating growth, and early metastasis ${ }^{21}$. Early detection of melanoma can contribute to a favorable prognosis and survival rate for melanoma patients. Unfortunately, current diagnostic methods are limited, so it is urgently needed to explore new sensitive and cost-effective biomarkers for the development of novel noninvasive tools ${ }^{22}$. Recently, more and more studies have suggested many biomarkers for the prognosis and diagnosis of melanoma patients, such as specific expression genes, methylation levels, serum miRNAs, and lncRNAs ${ }^{23,24}$. Among them, lncRNAs attract growing attention due to their high specificity, high sensitivity, and noninvasive characteristics.

Recent studies have demonstrated that several lncRNAs participate in the initiation and progression of melanoma via modulating important tumor-associated genes and various miRNAs ${ }^{25}$. Wang et al. ${ }^{26}$ suggested lncRNA CASC2 as a tumor suppressor due to its significant downregulation in melanoma patients and its overexpression suppressing the proliferation and metastasis of melanoma cells via sponging miR-181a.
A study from Jiao et al. ${ }^{27}$ showed that lncRNA LINC00963, an overexpressed lncRNA in melanoma, exhibited a tumor-promotive role by inhibiting the metastasis potential of melanoma cells via sponging miRNA-608 to increase NACC1 expression. Besides, several lncRNAs have been confirmed to serve as tumor biomarkers for the diagnosis and prognosis of melanoma patients ${ }^{28}$. These findings encouraged us to further explore more tumor-related lncRNAs. Recently, the distinct upregulation of LINC00173 was reported in several tumors and its tumor-promotive role was also confirmed in breast cancer and lung cancer ${ }^{17,19}$. However, the expression and clinical significance of LINC00173 in melanoma have not been investigated.

In this study, we identified a novel melanoma-related IncRNA, i.e., LINC00173, which was confirmed to be distinctly overexpressed in our cohort (163 patients). Also, we observed that the patients with advanced stages displayed a higher level of LINC00173, indicating that LINC00173 may contribute to the clinical progression of melanoma. Then, we performed ROC assays for the determination of the diagnostic value of LINC00173 expression, finding that high LINC00173 levels were robust in distinguishing melanoma tissues from 
normal melanoma specimens. In addition, the similar results were also observed when we further performed subgroup analysis based on the clinical stages of 163 melanoma patients. Moreover, clinical assays revealed that melanoma patients with higher LINC00173 expressions were associated with lymph node metastasis and advanced tumor stage, and suffered poorer OS and DFS, suggesting it may act as a tumor promotor in the clinical progress of melanoma patients. Finally, multivariate assays demonstrated high LINC00173 expression as an independent indicator of unfavorable OS and DFS of melanoma patients. Our findings may help the development of novel clinical tools in the early screening and the prediction of clinical prognosis before the clinical treatments.

Several limitations should be considered. First, the sample size was relatively small, and large clinical trials were needed to conduct. Second, replications in other groups were not

\section{REFERENCES}

1. Bray F, Ferlay J, Soerjomataram I, Siegel RL, Torre LA, Jemal A. Global cancer statistics 2018: GLOBOCAN estimates of incidence and mortality worldwide for 36 cancers in 185 countries. CA Cancer J Clin. 2018;68(6):394-424. https://doi.org/10.3322/caac.21492

2. Fu Q, Chen N, Ge C, Li R, Li Z, Zeng B, et al. Prognostic value of tumor-infiltrating lymphocytes in melanoma: a systematic review and meta-analysis. Oncoimmunology. 2019;8(7):1593806. https:// doi.org/10.1080/2162402x.2019.1593806

3. Innát P, Ostruszka P, Vávra P, Peteja M, Zonča P. Treatment strategies for patients with colorectal carcinomaand synchronous liver metastases. Rozhledy v chirurgii: mesicnik Ceskoslovenske chirurgicke spolecnosti. 97(10):451-4.

4. Mody K, Baldeo C, Bekaii-Saab T.Antiangiogenic therapy in colorectal cancer. Cancer J. 2018;24(4):165-70. https://doi.org/10.1097/ ppo.0000000000000328

5. Ikoma N, Raghav K, Chang G. An update on randomized clinical trials in metastatic colorectal carcinoma. Surg Oncol Clin N Am. 2017;26(4):667-87. https://doi.org/10.1016/j.soc.2017.05.007

6. Jathar S, Kumar V,Srivastava J, Tripathi V. Technological developments in IncRNA biology. Adv Exp Med Biol. 2017;1008:283-323. https:// doi.org/10.1007/978-981-10-5203-3_10

7. Jarroux J, MorillonA, PinskayaM. History, discovery, and classification of IncRNAs. Adv Exp Med Biol. 2017;1008:1-46. https://doi. org/10.1007/978-981-10-5203-3_1

8. Zhang Y, Tao Y, Liao Q. Long noncoding RNA: a crosslink in biological regulatory network. Brief Bioinform. 2018;19(5):930-45. https:// doi.org/10.1093/bib/bbx042

9. Kopp F, Mendell JT. Functional classification and experimental dissection of long noncoding RNAs. Cell. 2018;172(3):393-407. https://doi.org/10.1016/j.cell.2018.01.011

10. Salmena L, Poliseno L, Tay Y, Kats L, Pandolfi PP. A ceRNA hypothesis: the Rosetta Stone of a hidden RNA language? Cell. 2011;146(3):353-8. https://doi.org/10.1016/j.cell.2011.07.014

11. Renganathan A, Felley-Bosco E. Long noncoding RNAs in cancer and therapeutic potential. Adv Exp Med Biol. 2017;1008:199-222. https://doi.org/10.1007/978-981-10-5203-3_7 conducted. Finally, the potential function and molecular mechanisms of LINC00173 were not explored in melanoma.

\section{CONCLUSION}

Our study presented that LINC00173 expression was abnormally elevated in melanoma and may serve as a novel biomarker for predicting diagnosis and clinical progression of melanoma patients.

\section{AUTHORS' CONTRIBUTIONS}

MW, WL: Writing - original draft, Data curation, Formal Analysis. WL: Conceptualization, Data curation. CW: Conceptualization, Writing - review \& editing. MW, WL: contributed equally to the work.

12. Chandra Gupta S, Nandan Tripathi Y. Potential of long non-coding RNAs in cancer patients: from biomarkers to therapeutic targets. Int J Cancer. 2017;140(9):1955-67. https://doi.org/10.1002/ ijc.30546

13. Beermann J, Piccoli MT, Viereck J, Thum T. Non-coding RNAs in development and disease: background, mechanisms, and therapeutic approaches. Physiol Rev. 2016;96(4):1297-325. https://doi. org/10.1152/physrev.00041.2015

14. Han P, Li JW, Zhang BM, Lv JC, Li YM, Gu XY, et al. The IncRNA CRNDE promotes colorectal cancer cell proliferation and chemoresistance via miR-181a-5p-mediated regulation of Wnt/ beta-catenin signaling. Mol Cancer. 2017;16(1):9. https://doi. org/10.1186/s12943-017-0583-1

15. Botti G, Marra L, Malzone MG, Anniciello A, Botti C, Franco R, et al. LncRNA HOTAIR as prognostic circulating marker and potential therapeutic target in patients with tumor diseases. Curr Drug Targets. 2017;18(1):27-34. https://doi.org/10.2174/13894501 17666151209122950

16. Lu D, Luo P, Wang Q, Ye Y, Wang B. IncRNA PVT1 in cancer: a review and meta-analysis. Clin Chim Acta. 2017;474:1-7. https:// doi.org/10.1016/j.cca.2017.08.038

17. Yang Q, Tang Y, Tang C, Cong H, Wang X, Shen X, et al. Diminished LINC00173 expression induced miR-182-5paccumulation promotes cell proliferation, migration and apoptosis inhibition via AGER/ NF-kappaB pathway in non-small-cell lung cancer. Am J Transl Res. 2019;11(7):4248-62. PMID: 31396332

18. Mao Y, Fu Z, Zhang Y, Dong L, Zhang Y, Zhang Q, et al. A sevenIncRNA signature predicts overall survival in esophageal squamous cell carcinoma. Sci Rep. 2018;8(1):8823. https://doi.org/10.1038/ s41598-018-27307-2

19. Fan H, Yuan J, Li X, Ma Y, Wang X, Xu B, et al. LncRNA LINC00173 enhances triple-negative breast cancer progression by suppressing miR-490-3p expression. Biomed Pharmacother. 2020;125:109987. https://doi.org/10.1016/j.biopha.2020.109987

20. Hu Z, Yang D, Tang Y, Zhang X, Wei Z, Fu H, et al. Five-long non-coding RNA risk score system for the effective prediction of gastric cancer patient survival. Oncol Lett. 2019;17(5):4474-86. https://doi.org/10.3892/ol.2019.10124 
21. Ingraffea A. Melanoma. Facial Plast Surg Clin N Am. 2013;21(1):3342. https://doi.org/10.1016/j.fsc.2012.11.007

22. Lim SY, Lee JH, Diefenbach RJ, Kefford RF, Rizos H. Liquid biomarkers in melanoma: detection and discovery. Mol Cancer. 2018;17(1):8. https://doi.org/10.1186/s12943-018-0757-5

23. Micevic G, Theodosakis N, Bosenberg M. Aberrant DNA methylation in melanoma: biomarker and therapeutic opportunities. Clin Epigenet. 2017;9:34.https://doi.org/10.1186/s13148-017-0332-8

24. Darmawan CC, Jo G, Montenegro SE, Kwak Y, Cheol L, Cho $\mathrm{KH}$, et al. Early detection of acral melanoma: a review of clinical, dermoscopic, histopathologic, and molecular characteristics. J Am Acad Dermatol. 2019;81(3):805-12. https://doi.org/10.1016/j. jaad.2019.01.081
25. Li J, Li Z, Zheng W, Li X, Wang Z, Cui Y, et al. LncRNA-ATB: an indispensable cancer-related long noncoding RNA. Cell Prolif. 2017;50(6):e12381. https://doi.org/10.1111/cpr.12381

26. Wang Z, Wang $X$, Zhou H, Dan X, Jiang L, Wu Y. Long non-coding RNA CASC2 inhibits tumorigenesis via the miR-181a/PLXNC1 axis in melanoma. Acta Biochim Biophys Sin (Shanghai). 2018;50(3):26372. https://doi.org/10.1093/abbs/gm×148

27. Jiao H, Jiang S, Wang H, Li Y, Zhang W. Upregulation of LINC00963 facilitates melanoma progression through miR-608/NACC1 pathway and predicts poor prognosis. Biochem Biophys Res Commun. 2018;504(1):34-9. https://doi.org/10.1016/j.bbrc.2018.08.115

28. Bolha L, Ravnik-Glavač M, Glavač D. Long noncoding RNAs as biomarkers in cancer. Dis Markers. 2017;2017:7243968. https:// doi.org/10.1155/2017/7243968 